\title{
Physics and applications of photonic crystals
}

\author{
Ekmel Ozbay ${ }^{\mathrm{a}, \mathrm{b}, *}$, Irfan Bulu ${ }^{\mathrm{a}}$, Koray Aydin ${ }^{\mathrm{a}}$, \\ Humeyra Caglayan $^{\mathrm{a}}$, Kaan Guven ${ }^{\mathrm{a}}$ \\ ${ }^{a}$ Department of Physics, Bilkent University, Bilkent, 06800 Ankara, Turkey \\ ${ }^{\mathrm{b}}$ Nanotechnology Research Laboratory, Bilkent University, Bilkent, 06800 Ankara, Turkey
}

Received 12 June 2004; received in revised form 22 July 2004; accepted 2 August 2004

Available online 26 August 2004

\begin{abstract}
In this article, we investigate how the photonic band gaps and the variety of band dispersions of photonic crystals can be utilized for various applications and how they further give rise to completely novel optical phenomena. The enhancement of spontaneous emission through coupled cavity waveguides in a one-dimensional silicon nitride photonic microcrystal is investigated. We then present the highly directive radiation from sources embedded in two-dimensional photonic crystals. The manifestation of novel and intriguing optical properties of photonic crystals are exemplified experimentally by the negative refraction and the focusing of electromagnetic waves through a photonic crystal slab with subwavelength resolution.
\end{abstract}

(C) 2004 Elsevier B.V. All rights reserved.

PACS: 42.70.Qs; 42.60.Da; 42.82.Et; 78.20.Ci

Keywords: Photonic crystal; Coupled cavity waveguide; Spontaneous emission; Negative refraction; Left-handed material

\section{Introduction}

It is amazing that a simple analogy between an electron in a semiconductor crystal (periodic arrangement of atomic potentials) and a photon in a periodically modulated dielectric medium can reveal so enormously rich and novel electromagnetic (EM) phenomena [1,2]. Photonic crystals (PCs), as we call them now, are periodic dielectric, or metallic structures, which possess a variety of band disper-

\footnotetext{
* Corresponding author. Tel.: +90 312 2901966; fax: +90 3122664579

E-mail address: ozbay@fen.bilkent.edu.tr (E. Ozbay).
}

sions, and band gaps, where the propagation is prohibited for certain ranges of wavelengths. Using different materials (i.e., different dielectric constants) and by adjusting geometrical parameters, the propagation of light can be modified virtually in any way in a controllable manner. Furthermore, the scale invariant nature of the governing Maxwell's equations enables the study of electromagnetic phenomena in the first place, without being held back by structural complexities. A similar advantage is also present from a dimensionality point of view; that is, the lack of confinement in spatial directions in 2D or 1D PC structures does not hinder the investigation of certain EM phenomena. Having said this, keeping the 
structural uniformity while scaling the PCs down to optical wavelengths is still a challenging problem, especially in three-dimensions (3D). Numerous fabrication techniques are investigated in the last decade, such as alternating layer deposition and etching process for 3D PCs [3], and electron lithography in combination with dry etching for 2D AlGaAs PCs [4]. The investigation of PCs was quickly spread over a wide range of photonics applications in the last decade, gearing towards micro- and nanoscale to be used in telecommunication and optical wavelengths [5-9].

In this paper, we aim to provide a brief review of certain physical properties and applications of PCs. The paper is organized as follows: First, we consider the optical properties of coupled micro-cavity waveguide $(\mathrm{CMCW})$ structures in 1D PCs and the enhancement of the spontaneous emission. Next, we discuss how a 2D PC can be utilized to obtain a highly directive radiation from embedded sources. The last section is devoted to the experimental demonstration and simulation of a completely novel optical phenomenon, namely the negative refraction, and subwavelength focusing through a 2D PC slab.

\section{Coupled cavity waveguides in 1D photonic microcrystals and the enhancement of spontaneous emission}

Many optical applications demand the ability to control the spontaneous emission for inhibition or enhancement. Fermi's Golden Rule states that the spontaneous emission rate is directly proportional to the photon density of modes, $\Gamma_{\mathrm{s}} \sim \rho(\omega) \sim v_{\mathrm{g}}^{-1}$. By introducing a defect into a PC structure, a highly localized mode inside the photonic band gap appears, where the density of photon modes can be altered locally $[10,11]$, analogous to the impurity states in a semiconductor band gap [12]. The modification of spontaneous emission from atoms placed in 1D photonic band gap structures has been demonstrated $[13,14]$.

Here, we present the enhancement and guiding of light through a coupled micro-cavity (CMC) structure consisting of $\mathrm{Si}_{3} \mathrm{~N}_{4} / \mathrm{SiO}_{2}$ (silicon nitride/siliconoxide) layers with $\lambda / 2 \quad \mathrm{SiO}_{2}$ cavity layers using photoluminescence (PL) spectra measurements (Fig. 1). The layers were deposited on glass substrates

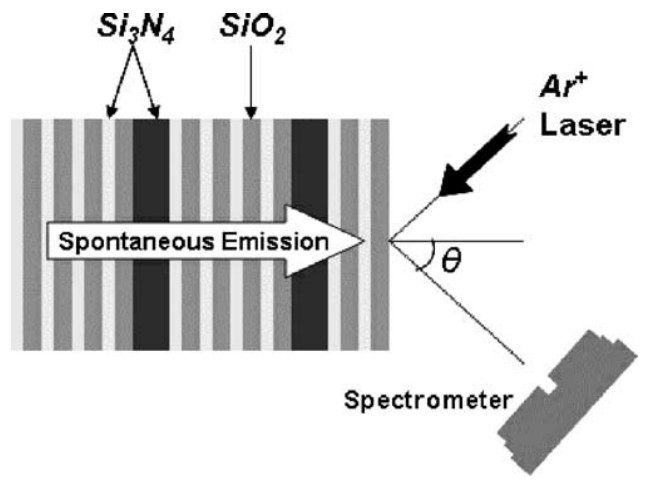

Fig. 1. Schematic of the coupled micro-cavity structure and the experimental setup for measuring the photoluminescence spectra.

by plasma enhanced chemical vapor deposition (PECVD) at $250{ }^{\circ} \mathrm{C}$ [14]. The cavities are introduced by doubling the deposition time of the silicon-oxide layers. The thicknesses are chosen as $d_{\mathrm{L}}=100 \mathrm{~nm}$ and $d_{\mathrm{H}}=70 \mathrm{~nm}$ for $\mathrm{SiO}_{2}$ and $\mathrm{Si}_{3} \mathrm{~N}_{4}$ layers and $200 \mathrm{~nm}$ for the cavity layers. The refractive indices and thicknesses of layers were $n_{\mathrm{SiO}_{2}}=1.46, n_{\mathrm{Si}_{3} \mathrm{~N}_{4}}=1.98, d_{\mathrm{SiO}_{2}}=$ $124.8 \mathrm{~nm}$, and $d_{\mathrm{Si}_{3} \mathrm{~N}_{4}}=92.0 \mathrm{~nm}$. The $\lambda / 2 d_{\text {cavity }}=$ $184 \mathrm{~nm}$ cavities were deposited with an intercavity distance $\Lambda=4.5$ pairs. The room temperature PL measurements were performed using a $1-\mathrm{m}$ double monochromator with a spectral resolution of $2 \mathrm{~nm}$ and an $\mathrm{Ar}^{+}$laser operating at $488 \mathrm{~nm}$ with $120 \mathrm{~mW}$ output power. The transmission spectrumisobtained by afiber spectrometer.

Fig. 2a shows the transmission spectrum of a CMC structure with $36 \mathrm{Si}_{3} \mathrm{~N}_{4} / \mathrm{SiO}_{2}$ pairs and four $\mathrm{Si}_{3} \mathrm{~N}_{4}$ cavity layers. Nearly full transmission is obtained throughout the CMC band in agreement with transfer matrix calculations. The PL spectrum in Fig. 2b shows an overall enhancement of the spontaneous emission across the CMC band from 690 to $770 \mathrm{~nm}$, particularly at the lower band edge, compared to PL spectrum of a single $\mathrm{Si}_{3} \mathrm{~N}_{4}$ layer. Similar observations were reported $[15,16]$.

Theoretical analysis of CMCs is formulated within the framework of tight binding (TB) approximation [17-21]. Within the TB formalism, the dispersion relation of the CMC is given by $\omega_{k}=\Omega[1+\kappa \cos (k \Lambda)]$, where $\Omega$ is the single-cavity resonance frequency, $\kappa$ the TB coupling constant, and $\Lambda$ is the distance between adjacent cavities. For a CMC structure, $\kappa$ can be calculated from $\kappa=\Delta \omega / 2 \Omega$ 


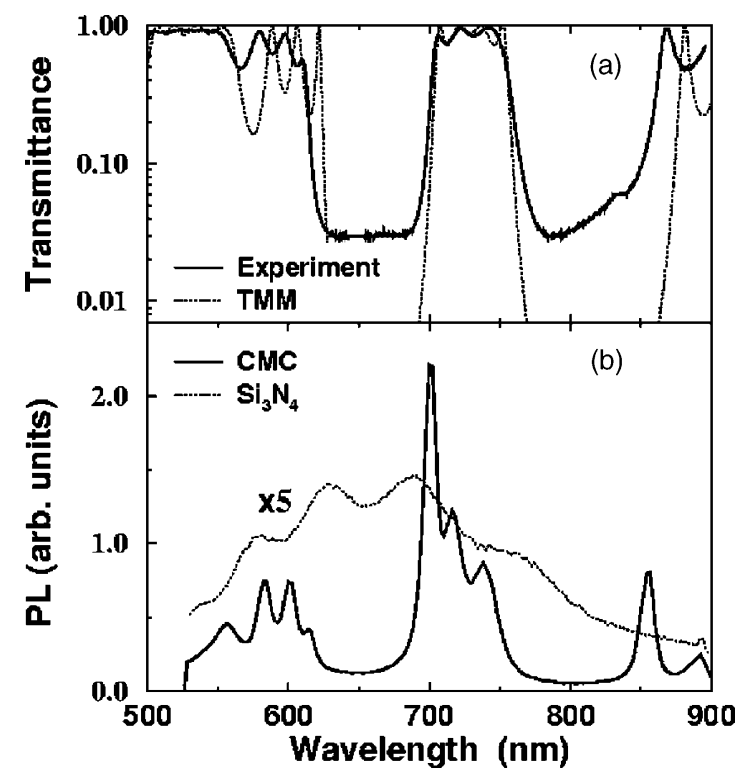

Fig. 2. (a) Comparison of the measured (solid line) and calculated (dotted line) transmission through the $\mathrm{Si}_{3} \mathrm{~N}_{4} / \mathrm{SiO}_{2}$ coupled microcavity structure. (b) Measured photoluminescence spectrum of the CMC structure. The $5 \times$ magnified thin curve denotes the PL spectrum of single $\mathrm{Si}_{3} \mathrm{~N}_{4}$ layer.

by measuring the single cavity resonance $\Omega$, and the bandwidth of the waveguide $\Delta \omega$. The group velocity and lifetime of photons follows from this relation as $v_{\mathrm{g}}=\nabla_{k} \omega_{k}=-\kappa \Lambda \Omega \sin (k \Lambda), \tau_{\mathrm{p}}=\left(L / v_{\mathrm{g}}\right)+(2 \pi L / c)$, where $L$ is the total length of the structure. Fig. 3 shows the calculated parameters using $\kappa$ determined experimentally. We stress that $v_{\mathrm{g}}$ at the guiding band center is one order of magnitude smaller than that in vacuum, and decreases further at the edges. Since smaller group velocity enhances optical processes, this property, combined with efficient transmission through coupled cavity waveguides, brings important advantages to optical applications. The position and width of the guiding band can be set by changing the thickness of the cavity layers and the intercavity distance accordingly.

\section{Highly directive radiation from photonic crystals hosting embedded sources}

In this section, we present an experimental and theoretical study of the angular distribution of the radiation emitted from a source embedded in a 2D PC. The spatial confinement of the radiation emitted by sources is highly desirable for certain antenna applications. Several studies are reported, which employ PCs for the confinement of the radiation as a cover to the source [22-24], or the source being embedded inside the PC $[25,26]$. By adjusting the geometrical parameters of the PC and thus the band structure, it is possible to confine the emitted power to a very narrow region at certain frequencies. We also show that the size of the PC is a critical parameter.

The photonic crystal in this study is a $2 \mathrm{D}$ square array of cylindrical alumina rods of radius $1.55 \mathrm{~mm}$ and a dielectric constant of 9.61. The crystal period is $1.1 \mathrm{~cm}$. Band structure of the corresponding infinite $\mathrm{PC}$ for TM-polarized EM waves is given in Fig. 4. In the band structure we observe that at the minimum of the second band (upper band), only the modes along $\Gamma-X^{\prime}$ are allowed to propagate inside the PC, where $X^{\prime}$ represents all directions having the same symmetry properties of $X$ point. For the modes near the upper band edge, $k_{\|}=k_{x} \approx 0$. Apart from an additive

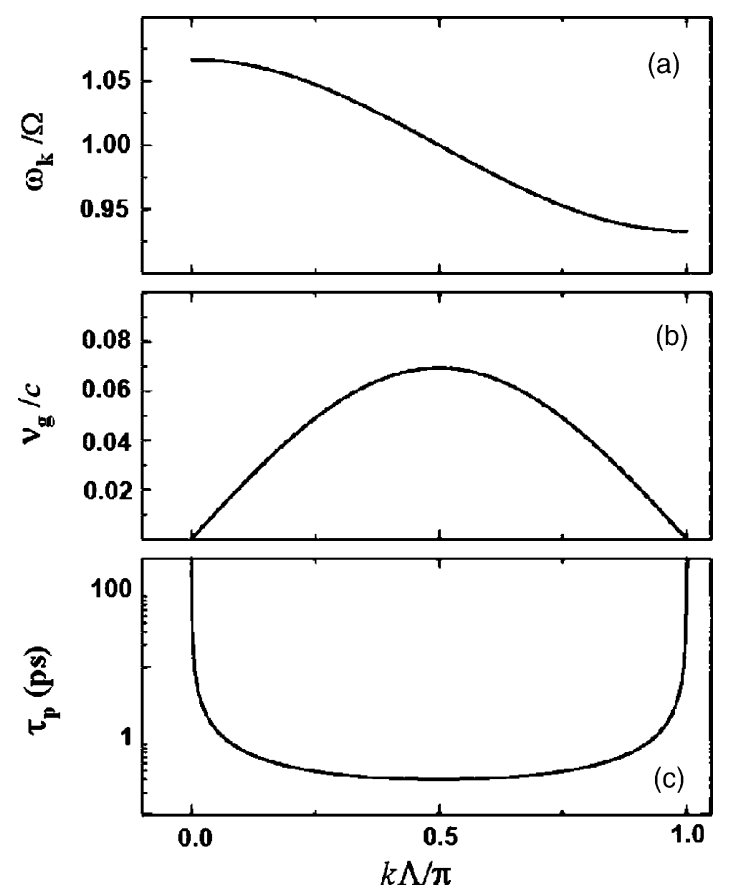

Fig. 3. (a) Calculated dispersion relation for a CMC sample using TB coupling parameter $\kappa \approx 0.067$. (b) Normalized group velocity and (c) photon lifetime as a function of wavevector $\boldsymbol{k}$. Notice that $v_{\mathbf{g}} \rightarrow 0$ and $\tau_{\mathrm{p}} \rightarrow \infty$ at the edges of the guiding band. 


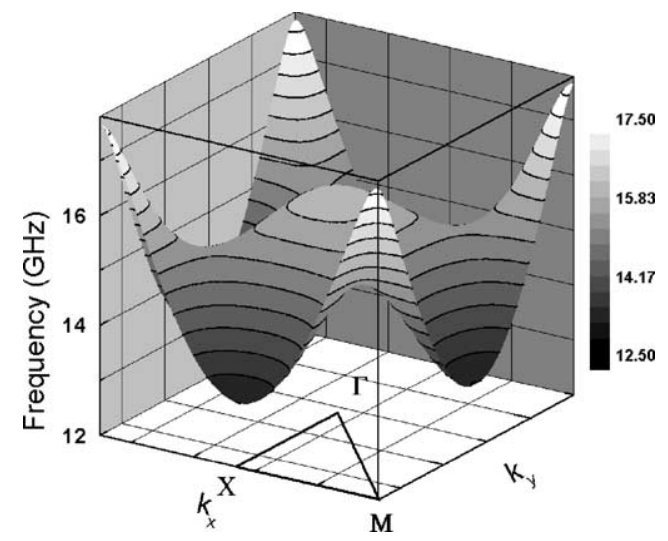

Fig. 4. The TM polarized second band of the PC in the first Brillouin zone.

reciprocal lattice vector, $k_{\|}$is conserved at the air-PC interface [27].

Hence, we conclude that for a source embedded inside the PC, operating at the upper band edge frequency, the emitted waves should be transmitted from the PC to air only along $\Gamma-X^{\prime}$ direction. In addition, the modes near the upper band edge are air modes, i.e., most of the energy of the EM waves is concentrated in the low dielectric material region as the waves propagate through the PC (Fig. 5a). Hence, when the waves near the upper band edge emerge from the surface of the PC, most of the power will flow through low dielectric material region of the PC (Fig. 5b). In conclusion, we can regard the exit points as radiation sources.

Delay time measurements of the present PC structure shown in Fig. 6a indicate that the group velocity near the upper band edge is reduced up to 22 times in comparison to air. Delay time is defined as $\tau_{\mathrm{p}}=\partial \varphi / \partial \omega$ where $\varphi$ is the difference between the phase of the EM wave inside the PC and in free space. Since at the upper band edge the modes are allowed to propagate only along $\Gamma-X^{\prime}$, these radiation sources will have a uniform distribution of phase differences. Therefore, the surface of the PC can be regarded as an antenna array operating at the upper band edge frequency with similar spatial and temporal distribution of power and a uniform phase difference. Consequently, the power radiating in the $\Gamma-X$ direction from the surface is anticipated to be highly confined.

The experimental observation of the confined radiation is performed by the setup depicted in

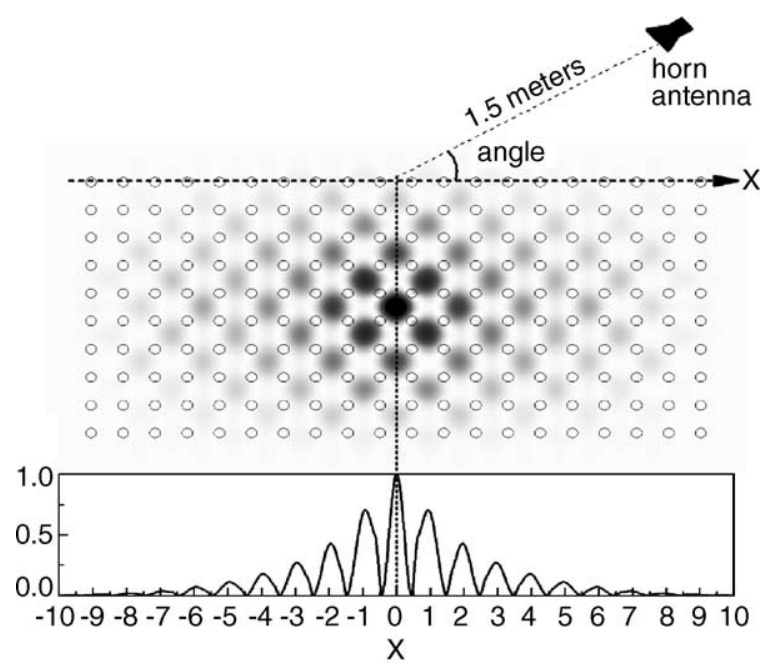

Fig. 5. (a) Experiment setup for a $2 \mathrm{D} 20 \times 10$ square array PC. The electric field intensity distribution of a source located at the center and operating at the second band edge frequency. Note the field is localized in between the dielectric rods (air). (b) Lateral distribution of the electric field intensity along the upper surface of the PC.
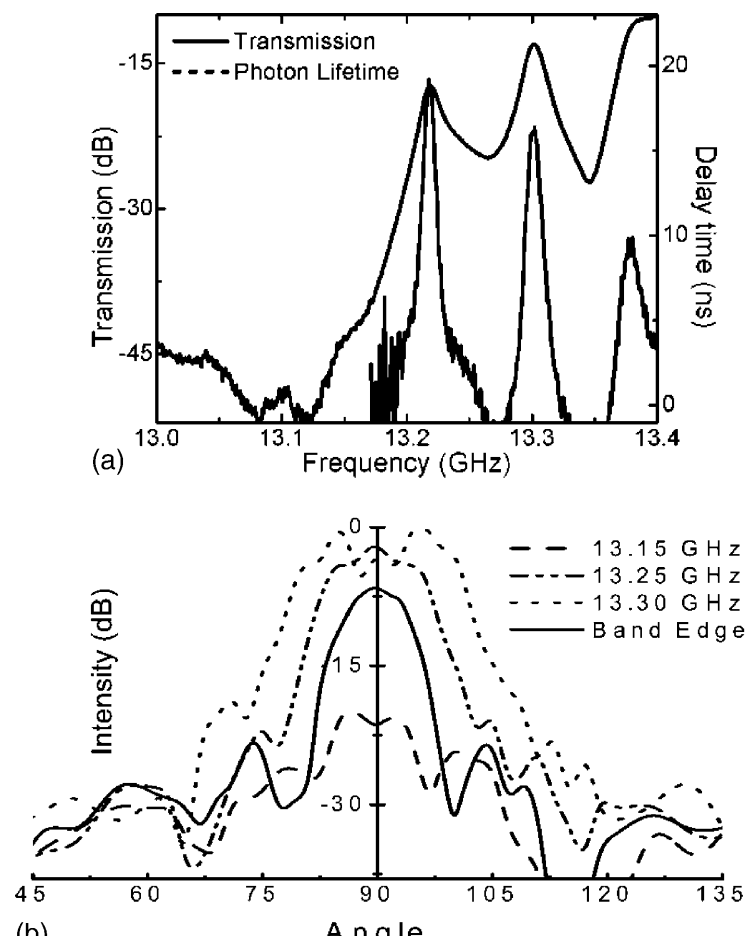

(b)

Angle

Fig. 6. (a) The transmission and delay time measured near the upper band edge of the PC. (b) The far field radiation patterns of various frequencies in the upper band emitted from the PC surface perpendicular to the $\Gamma X$ direction. 

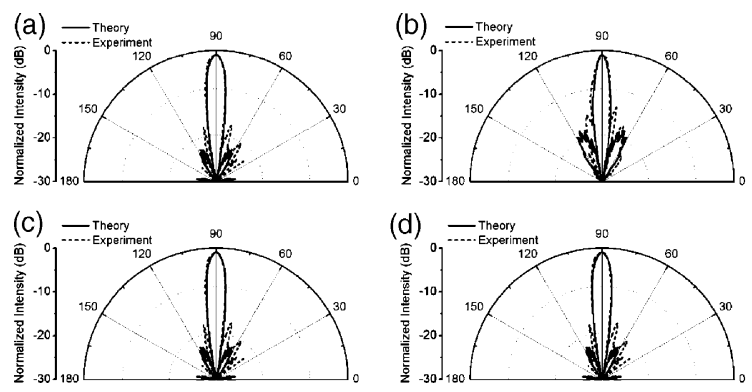

Fig. 7. Measured (dashed curves) and simulated (solid curves) far field patterns at the upper band edge frequency of $13.21 \mathrm{GHz}$ for different lateral lengths of the PC (a) $32 \times 16$, (b) $28 \times 16$, (c) $24 \times$ 16 , and (d) $20 \times 16$ layers.

Fig. 5a. A monopole antenna is inserted in to the $2 \mathrm{D}$ $\mathrm{PC}$ as an omnidirectional source. HP-8510C network analyzer is used to excite the monopole source and to measure the power emitted from the monopole source. The measured far field radiation patterns for a square array of $32 \times 20$ alumina rods at various frequencies near the upper band edge are presented in Fig. 6b. The minimum half power beam width is obtained at $13.21 \mathrm{GHz}$ and found to be $8^{\circ}$. The maximum delay time, as shown in Fig. 6a occurs at 13.21 GHz. Hence, we conclude that the upper band edge is at $13.21 \mathrm{GHz}$. It is evident that the angular distribution of power strongly depends on the radiation frequency. The radiation patterns spread quickly for frequencies lying deep in the upper band. This can be explained by the presence of equal frequency modes along different directions at these frequencies.

The measured and calculated far field radiation patterns for different $\mathrm{PC}$ lateral lengths are presented in Fig. 7. The measured half power beam widths show an optimum crystal length, which is found to be 24 layers, corresponding a beam width of $6^{\circ}$. To our knowledge, this is the minimum experimental value obtained from PC embedded sources reported so far. We observe that deviations from its optimum value affect both the radiation pattern and the half power beam widths. The change is more significant when the lateral length is decreased from its optimum value. This can be explained by the fact that the strength of the surface radiators decreases rapidly away from the center of the PC (Fig. 4b). Consequently, increasing the lateral length (adding more but weaker surface radiators) is less significant when compared to the effect of decreasing (removing stronger surface radiators).

\section{Negative refraction and point focusing using a photonic crystal superlens}

The previous two sections can be considered as examples of the improvement of existing optical processes by utilizing the optical properties of PCs. However, there is more that the PCs can provide. In this section, we consider some examples of the novel optical phenomena that have become accessible by the PCs.

In 1960, Veselago [28] proposed and investigated the electrodynamics in a medium possessing negative index of refraction. Almost four decades after its introduction, the realization idea of such media has been revived, albeit using artificial composite structures having negative permittivity and negative permeability, respectively [29-32], bringing controversial ideas along with it [33-35].

Meanwhile, several studies reported anomalous refraction at the PC interfaces called "super prism phenomenon" [36-38]. Following this, PCs made a surprising entry in the quest for the realization of negative index materials. Analysis of the band structures of PCs revealed that the underlying mechanism for the negative refraction in PCs is not unique. When the incident field couples to a convex PC band in $\boldsymbol{k}$-space, the "negative" curvature of the band causes the beam bend negatively [39,41], although the PC essentially remains to be a "positive index" medium. Alternatively, if the group velocity and phase velocity derived from the band dispersion are antiparallel for all values of $\boldsymbol{k}$, the effective index of refraction becomes negative [40]. Both mechanisms are confirmed experimentally $[41,42]$.

In this section, we review the experimental investigation of negative refraction and subwavelength focusing of microwaves through a 2D PC slab reported recently [41]. The PC consists of a square array of alumina rods in air with the dielectric constant of $\varepsilon=9.61$ and a diameter of $3.15 \mathrm{~mm}$. The PC lattice constant is $a=4.79 \mathrm{~mm}$. The transverse magnetic(TM) polarized valence band of the photonic crystal calculated by plane wave expansion method is shown in Fig. 8a. Following the analysis of Ref. [39], the scaled frequency range that gives negative refraction for the present structure extends from $\tilde{\omega}=0.2093$ to $\tilde{\omega}=0.24673(\tilde{\omega}=\omega a / 2 \pi c$, where $a$ is the PC period). The EFSs for air and PC at $\tilde{\omega}=0.2189$ are shown in 


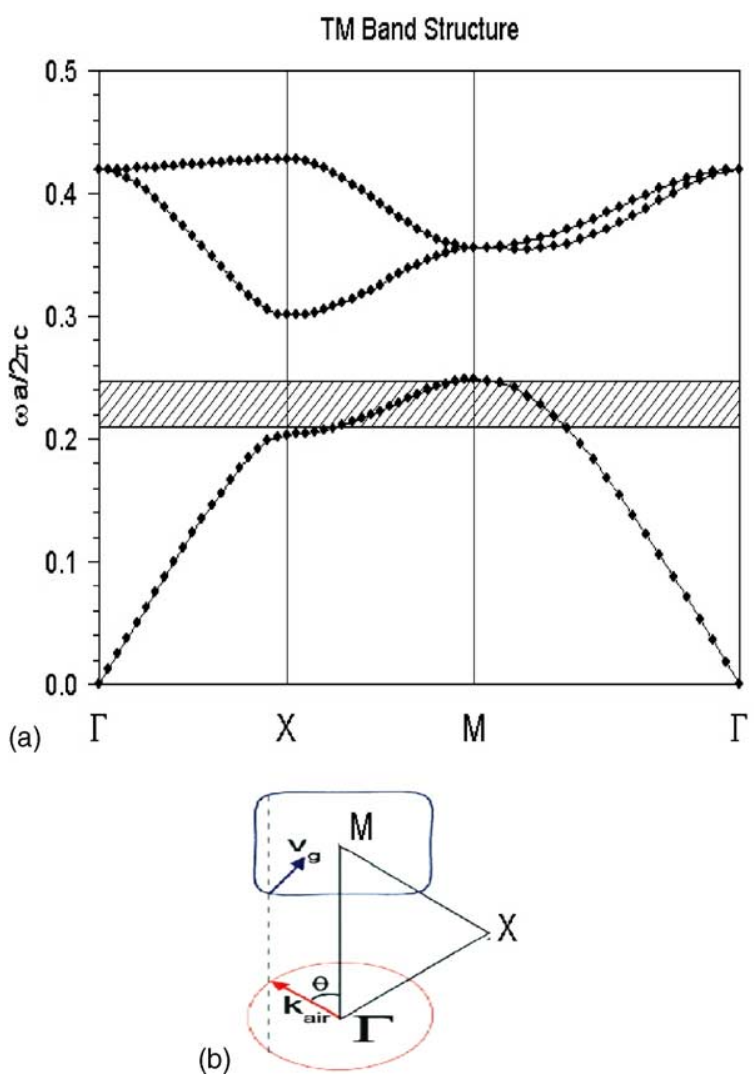

Fig. 8. (a) TM polarized band diagram of the PC. Negative refractive region is shaded. (b) Frequency contours of air (bottom) and PC (top) at $\omega a / 2 \pi c=0.2189(f=13.698 \mathrm{GHz}) . \theta$ denotes the incidence angle from air to PC.

Fig. 8b. We note that the conservation of surfaceparallel wave vector gives the direction of the refracted waves inside the $\mathrm{PC}$, as indicated in this figure.

The experimental setup consists of an HP 8510C network analyzer, a horn antenna as the transmitter, and a monopole antenna as the receiver (Fig. 9). Electromagnetic waves at frequency $\tilde{\omega}=0.2189$ are incident to the $\Gamma M$ interface at an angle of $45^{\circ}$. The spatial distribution of the time averaged field intensity along the air-PC and PC-air interface locations are measured first without the PC (i.e., freespace propagation), and then with the PC. In addition, the finite difference time domain (FDTD) method is used to simulate this experimental setup, in order to obtain the refracted beam profile. Fig. 10a shows the measured and simulated spatial distributions of

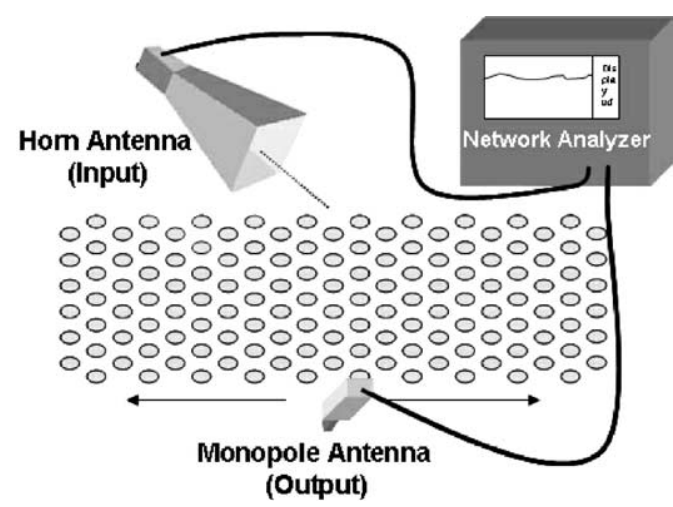

Fig. 9. Schematics of the experimental setup. In the refraction experiment, a transmitter horn antenna and a receiver monopole antenna are used. In the focusing effect experiment, both the transmitter and receiver are monopole antennas.
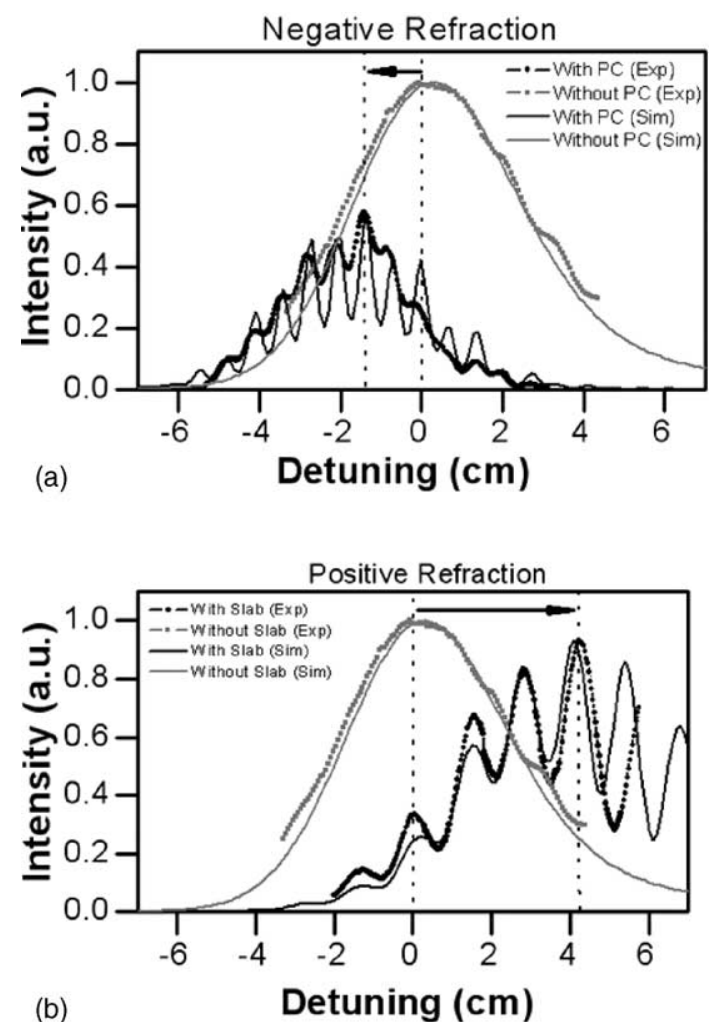

Fig. 10. Comparison between negative (top) and positive (bottom) refraction. (a) Grey dots (grey curve) denote the measured (simulated) average intensity at the at the air-PC interface without PC. Black dots (black curve) denote the measured (simulated) power at the PC-air interface. (b) Same as in (a) but using a slab of polystyrene pellets instead of PC. 
intensity at both interfaces. It is evident that the outgoing beam profile is shifted to the left (negative) side, relative to the incident beam profile. For comparison purposes, the refraction through a slab that contains only polystyrene pellets (refractive index of $n=1.46$ ) is shown in Fig. 10b.

For the selected frequency, the incident field couples to the first band and propagates according to its dispersion as a single beam, since $\lambda>2 \sqrt{2}$ (i.e., no Bragg reflections). Therefore, Snell's Law may be applied to the PC as $n_{\mathrm{eff}}\left(f, \boldsymbol{k}_{\mathbf{i}}\right) \sin \theta_{\mathrm{r}}=n_{\text {air }} \sin \theta_{\mathrm{i}}$, where $\theta_{\mathrm{i}}$ is the angle of incidence, $\theta_{\mathrm{r}}$ the angle of refraction, and $n_{\text {eff }}\left(f, \boldsymbol{k}_{\mathbf{i}}\right)$ is the frequency dependent, anisotropic refractive index along the propagation direction $\boldsymbol{k}_{\mathbf{i}}$. This yields $n_{\text {eff }}=-1.94$, which is very close to the value of -2.06 calculated by the FDTD method.

In the aforementioned frequency range the EFCs are square shaped around the $M$ point (Fig. 8b); hence the anisotropy of the refractive index is evident. This also restricts the angular range, where $\boldsymbol{v}_{\mathbf{g}} \boldsymbol{k}_{\mathbf{i} \|}<0$ (while $\left.\boldsymbol{v}_{\mathbf{g}} \boldsymbol{k}_{\mathbf{i}}>0\right), \boldsymbol{v}_{\mathbf{g}}=\nabla_{k} \omega(k)$ is the group velocity inside the PC, and $\boldsymbol{k}_{\mathbf{i} \|}$ is the component of $\boldsymbol{k}$ parallel to the interface. At $13.698 \mathrm{GHz}$, negative refraction is observed for $\theta_{\mathrm{i}}>20^{\circ}$ and the respective EFCs for air and for the PC have almost the same diameter, which maximizes the angular range of negative refraction for this structure. At higher frequencies, total internal reflection reduces the maximum incidence angle with negative refraction; whereas at lower frequencies the minimum angle where negative refraction occurs increases.

The presence of negative refraction raised a heated debate on the possibility of superlensing [43-45]. Ideally this required an isotropic index of refraction with a value of -1 . Anisotropy and deviations from negative unity will distort the focusing, but still exhibit unusual focusing properties. We tested the focusing properties of the present PC by a $15 \times 21$ layered slab structure. A monopole antenna is used as the point source $0.7 \mathrm{~mm}$ away from the air-PC interface, and the power distribution at the image plane $(0.7 \mathrm{~mm}$ away from PC-air interface) is scanned. The full width at half maximum (FWHM) of the focused beam is found to be $0.21 \lambda$, which is in good agreement with simulation (Fig. 11). The calculated FWHM of the beam at this plane in the absence of the PC is found to be $5.94 \lambda$, which implies a $25 \times$ enhancement compared to the free space case.

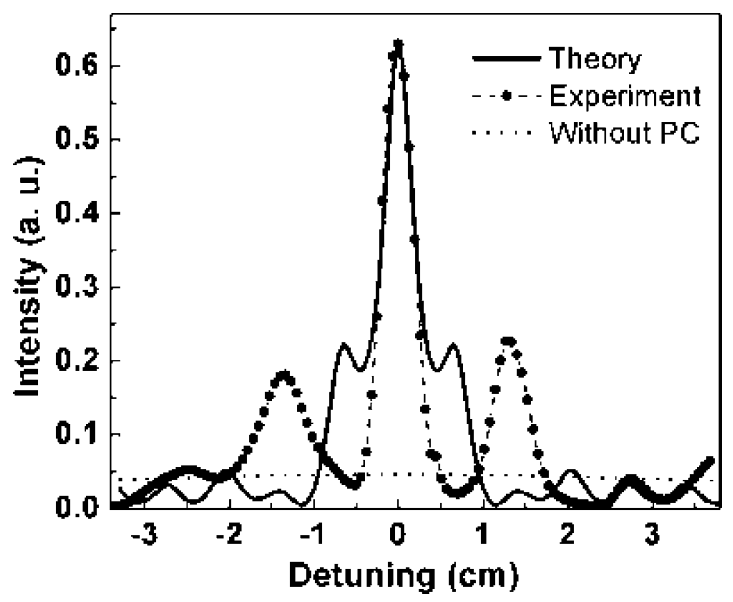

Fig. 11. Focusing: Measured power distribution (dots) and calculated average intensity (solid curve) at the image plane. Full width at half maximum of the measured image is $\sim 0.21 \lambda$. Spatial power distribution without PC is also shown (dotted thin line).

To investigate the subwavelength resolution, the same PC is used first with two coherent sources at $13.698 \mathrm{GHz}$ and then with two incoherent sources driven by separate signal generators operating at 13.698 and $13.608 \mathrm{GHz}$, respectively, to exclude interference effects. In both cases, the sources are separated by a distance of $\approx \lambda / 3$ and placed $0.7 \mathrm{~mm}$ away from the photonic crystal interface. Fig. 12 shows the lateral intensity profile in the image plane $0.7 \mathrm{~mm}$ away from the PC-air interface. In both cases, the source pair is clearly resolved. In contrast, no feature indicating source positions is visible in the absence of the PC. One can argue that the observed enhanced resolution can be attributed to the high refractive index as in the case of oil (or solid) immersion microscopy, where higher wave vectors, evanescent in air, can transmit through the crystal and form a near-field image with subwavelength resolution. To test this, we simulated two dielectric slabs of $n=3.1$ and $n=15$, respectively, for the incoherent source pair. We note that $n=3.1$ is also the refractive index of the alumina rods used in constructing the PC. Even with impractically high refractive index $(n=15)$, subwavelength resolution is not present (dashed lines in Fig. 12b). Besides, the large reflection due to high index contrast at the interface significantly reduces the transmitted power, when compared to the high (63\%) transmission obtained from the $\mathrm{PC}$ at this frequency. So, even if the observed subwavelength is associated 


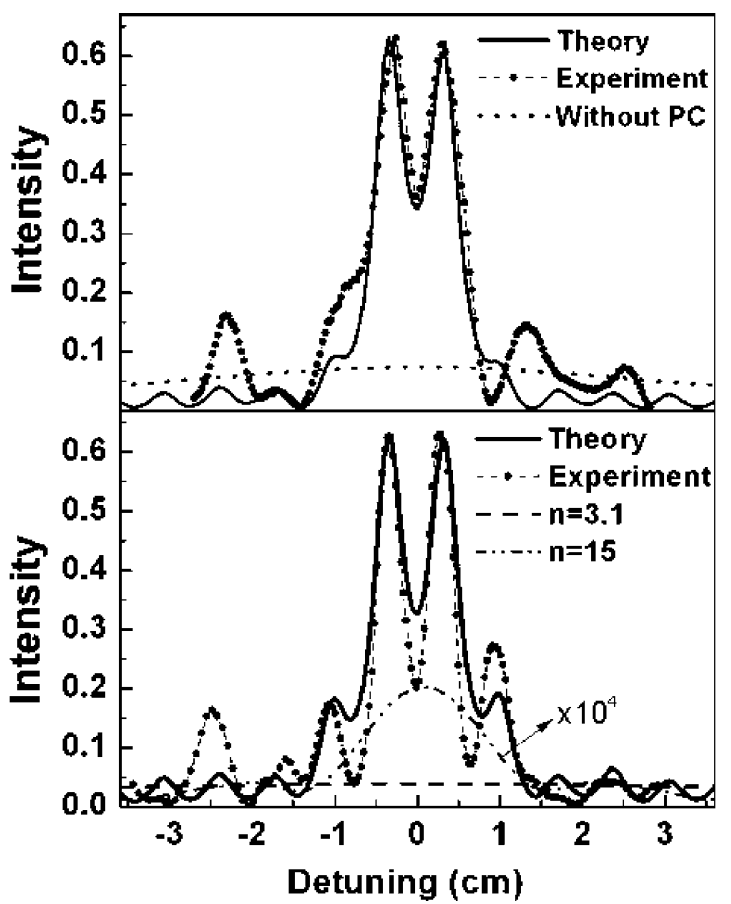

Fig. 12. (a) Measured (dots) and simulated (solid curve) power distribution at $0.7 \mathrm{~mm}$ away from the PC-air interface for two coherent sources separated by $\approx \lambda / 3$ at $0.7 \mathrm{~mm}$ away from the air-PC interface. Dotted line denotes the simulated intensity profile in the absence of PC. (b) Same setup with two incoherent point sources. The lower dashed and dot-dashed curves indicate the power distribution for uniform dielectric slabs with refractive indices $n=3.1$ and $n=15$, respectively. Note the magnification of the curve.

with near field effects, this is not achievable by ubiquitous materials.

The negative refraction and focusing effects reported here depend solely on the dielectric constant of the materials and on the geometrical parameters of the photonic crystal. Using transparent semiconductors with refractive indices similar to that presented here, it may be possible to observe these effects in optical wavelengths just by scaling the structures.

\section{Conclusions}

In this article we presented certain optical properties of PC structures. The improvement of existing optical applications by PCs is exemplified by the enhancement of spontaneous emission through a PC- based coupled micro-cavity waveguide, and by the exceptionally directive radiation from an omnidirectional source embedded in a PC. We then discussed a novel and unusual optical phenomenon observed in PCs, namely the negative refraction. This phenomenon may lead to optical applications such as flat lenses with subwavelength focusing abilities. These examples also demonstrated that the utilization of photonic crystals can be scaled down or up across the entire electromagnetic spectrum, thus making them potentially available for a wide range of applications. In summary, PC structures are anticipated to be an essential component of photonic integrated circuits in the near future.

\section{References}

[1] J.D. Joannopoulos, R.D. Meade, J.N. Winn, Photonic Crystals: Molding the Flow of Light, Princeton University Press, Princeton, NJ, 1995.

[2] C.M. Soukoulis (Ed.), Photonic Crystals and Light Localization in the 21st Century, Kluwer, Dortrecht, 2000.

[3] E. Kuramochi, et al. Opt. Quant. Elec. 34 (2002) 53.

[4] Y. Sugimoto, N. Ikeda, N. Carlsson, K. Asakawa, N. Kawai, K. Inoue, J. Appl. Phys. 91 (2002) 922.

[5] H. Benisty, C. Weisbuch, D. Labilloy, M. Rattier, C.J.M. Smith, T.F. Krauss, R.M. De La Rue, U. Houdré, C. Oesterle, D. Jouanin, Cassagne, J. Lightwave Tech. 17 (1999) 2063.

[6] T. Krauss, Y.P. Song, S. Thomson, C.D.W. Wilkinson, R.M. De La Rue, Electron. Lett. 30 (1994) 1444.

[7] P.L. Gourley, J.R. Wendt, G.A. Vawter, T.M. Brennan, B.E. Hammons, Appl. Phys. Lett. 64 (1994) 687.

[8] S. Noda, M. Imada, S. Ogawa, M. Mochizuki, A. Chutinan, IEEE J. Quant. Elec. 38 (2002) 726.

[9] J.M. Lourtioz, H. Benisty, A. Chelnokov, S. David, S. Olivier, Ann. Telecommunications 58 (2003) 1197.

[10] E. Yablonovitch, Phys. Rev. Lett. 58 (1987) 2059.

[11] A.M. Vredenberg, N.E.J. Hunt, E.F. Schubert, D.C. Jacobson, J.M. Poate, G.J. Zydzik, Phys. Rev. Lett. 71 (1993) 517.

[12] E. Yablonovitch, T.J. Gmitter, R.D. Meade, A.M. Rappe, K.D. Brommer, J.D. Joannopoulos, Phys. Rev. Lett. 67 (1991) 3380.

[13] M.D. Tocci, M. Scalora, M.J. Bloemer, J.P. Dowling, C.M. Bowden, Phys. Rev. A 53 (1996) 2799.

[14] M. Bayindir, S. Tanriseven, A. Aydinli, E. Ozbay, Appl. Phys. A 73 (2001) 125; M. Bayindir, S. Tanriseven, E. Ozbay, Appl. Phys. A 72 (2001) 117.

[15] F. Giorgis, Appl. Phys. Lett. 77 (2000) 522.

[16] A. Serpenguzel, S. Tanriseven, Appl. Phys. Lett. 78 (2000) 1388.

[17] C.M. De Sterke, Phys. Rev. E 57 (1998) 3502. 
[18] E. Lidorikis, M.M. Sigalas, E.N. Economou, C.M. Soukoulis, Phys. Rev. Lett. 81 (1998) 1405.

[19] A. Yariv, Y. Xu, R.K. Lee, A. Scherer, Opt. Lett. 24 (1999) 711.

[20] M. Bayindir, B. Temelkuran, E. Ozbay, Phys. Rev. Lett. 84 (2000) 2140.

[21] M. Bayindir, B. Temelkuran, E. Ozbay, Phys. Rev. B 61 (2000) R11855.

[22] E.R. Brown, C.D. Parker, E. Yablonovitch, J. Opt. Soc. Am. B 61 (1993) 404.

[23] R. Gonzalo, P. de Maagt, M. Sorolla, IEEE Trans. Microwave Theory Tech. 47 (1999) 2131.

[24] M. Qiu, S. He, Microwave Opt. Technol. Lett. 30 (2001) 41.

[25] S. Enoch, B. Gralak, E. Tayeb, Appl. Phys. Lett. 81 (2002) 1588.

[26] R. Biswas, E. Ozbay, B. Temelkuran, M. Bayindir, M.M. Sigalas, K.-M. Ho, J. Opt. Soc. Am. B 18 (2001) 1684.

[27] K. Sakoda, Optical Properties of Photonic Crystals, SpringerVerlag, Germany, 2001.

[28] V.G. Veselago, Soviet Phys. Uspekhi 10 (1968) 509.

[29] D.R. Smith, W.J. Padilla, D.C. Vier, S.C. Nemat-Nasser, S. Schultz, Phys. Rev. Lett. 84 (2000) 4184.

[30] R.A. Shelby, D.R. Smith, S. Schultz, Science 292 (2001) 77.

[31] D.R. Smith, S. Schultz, P. Markos, C.M. Soukoulis, Phys. Rev. B 65 (2002) 195104.

[32] M. Bayindir, K. Aydin, E. Ozbay, P. Markos, C.M. Soukoulis, Appl. Phys. Lett. 81 (2002) 120.

[33] R.W. Ziolkowski, E. Heyman, Phys. Rev. B 64 (2001) 056625.
[34] P.M. Valanju, R.M. Walser, A.P. Valanju, Phys. Rev. Lett. 88 (2002) 187401.

[35] A.L. Pokrovsky, A.L. Efros, Phys. Rev. Lett. 89 (2002) 093901.

[36] H. Kosaka, T. Kawashima, A. Tomita, M. Notomi, T. Tamamura, T. Sato, S. Kawakami, Phys. Rev. B 58 (1998) R10096.

[37] M. Notomi, Phys. Rev. B 62 (2000) 10696.

[38] B. Gralak, S. Enoch, G. Tayeb, J. Opt. Soc. Am. A 17 (2000) 1012.

[39] C. Luo, S.G. Johnson, J.D. Joannopoulos, J.B. Pendry, Phys. Rev. B 65 (2002) R201104.

[40] S. Foteinopoulou, E.N. Economou, C.M. Soukoulis, Phys. Rev. Lett. 90 (2003) 107402;

S. Foteinopoulou, C.M. Soukoulis, Phys. Rev. B 67 (2003) 235107.

[41] E. Cubukcu, K. Aydin, E. Ozbay, S. Foteinopoulou, C.M. Soukoulis, Nature 423 (2003) 604;

E. Cubukcu, K. Aydin, E. Ozbay, S. Foteinopoulou, C.M. Soukoulis, Phys. Rev. Lett. 91 (2003) 207401.

[42] P.V. Parimi, T.W. Lu, P. Vodo, S. Sridhar, Nature 426 (2003) 404.

[43] J.B. Pendry, Phys. Rev. Lett. 85 (2000) 3966.

[44] N. Garcia, M. Nieto-Vesperinas, Phys. Rev. Lett. 88 (2002) 207403.

[45] C. Luo, S.G. Johnson, J.D. Joannopoulos, Phys. Rev. B 68 (2003) 045115. 